\title{
EL SABER DEL DISCURSO HEGEMÓNICO COMO MANIPULADOR DE LAS MENTES $Y$ FORMADOR DE IDENTIDADES- LA REALIDAD DE LA IMPLEMENTACIÓN DEL PORTUGUÉS EN ZONA DE FRONTERA-
}

\author{
ROSÁRIO ACUÑA \\ Centro Regional de Profesores del Norte \\ RIVERA, URUGUAY \\ E-MAIL: rosmaf@adinet.com.uy
}


O "SABER" DO DISCURSO HEGEMÔNICO COMO MANIPULADOR DAS MENTES E FORMADOR DE IDENTIDADES- A REALIDADE DA IMPLEMENTAÇÃO DO PORTUGUÊS EM REGIÃO DE FRONTEIRA-

Resumo: Este trabalho reconhece que existe um discurso "oficial" que é transmitido à sociedade e que é assumido como verdadeiro e inquestionável. A região de fronteira é uma paisagem linguística complexa e em constante evolução na qual coexistem duas línguas-culturas que afetam a todas as áreas do quefazer social. Considera-se que as identidades, nesta região, estão claramente marcadas por estes discursos e representações, constituindo-se como parte do ideário comum. Palavras chave: Discursos hegemônicos; Identidades; Políticas Educativas; Políticas Linguísticas

\section{EL "SABER" DEL DISCURSO HEGEMÓNICO COMO MANIPULADOR DE LAS MENTES Y FORMADOR DE IDENTIDADES- LA REALIDAD DE LA IMPLEMENTACIÓN DEL PORTUGUÉS EN ZONA DE FRONTERA-}

Resumen: En este trabajo se reconoce que existe un discurso hegemónico que es impartido a la sociedad y que es asumido como verdadero e incuestionable. El contexto de frontera es un paisaje lingüístico complejo y en constante evolución en el cual coexisten dos lenguas- culturas que afectan a todas las áreas del quehacer social. Se considera que las identidades en esta región están claramente marcadas por estos discursos y representaciones, constituyéndose como parte del ideario común. Palabras clave: Discursos hegemónicos; Identidades; Políticas Educativas; Políticas Lingüísticas

THE “KNOWLEDGE" OF THE HEGEMONIC DISCOURSE AS A MANIPULATOR OF THE MINDS AND TRAINER OF IDENTITIES--THE REALITY OF THE IMPLEMENTATION OF THE PORTUGUESE IN BORDER AREA -

Abstract: This paper recognizes that there is a hegemonic discourse that is taught to the society and which is taken as true and unquestionable. Border context is a linguistic landscape complex and constantly evolving in which coexist two languages - cultures affecting all areas of social life. Identities in this region are considered clearly marked by these discourses and representations, becoming part of the common ideology. Keywords: Hegemonic discourses-; Identities; Educational Policies; Linguistic Policies 


\section{INTRODUCCIÓN}

La educación se encuentra en un momento donde es incuestionable la necesidad de búsqueda de un principio y /o objetivos que sean apropiados considerando el tipo de sociedad que se quiere construir y el tipo de hombre que se desea formar. Esto podría suponer la formación de nuevas bases para la política, donde las masas (gobernados) sean capaces de cuestionar y actuar de forma crítica. Para ello se tendría que considerar una escuela que sufra una profunda transformación que trascienda la posibilidad de una efectiva universalización de la enseñanza, se hace necesario el cambio de las orientaciones cognitivas y del contenido comunicativo de la escuela.

Los programas y centros de enseñanza son probablemente, junto con la familia y los medios de comunicación, la fuente más importante de adquisición de conocimientos, actitudes, creencias y normas, ejerciendo una influencia fundamental en las posturas adoptadas por los individuos frente a cuestiones sociales primordiales como el trabajo, el inconformismo y los conflictos dentro de una sociedad, la comprensión internacional, la eficacia personal como ciudadano y las aspiraciones de cada cual sobre su porvenir. Es característica de la mayoría de las escuelas el socializar y formar bajo las pautas ya existentes y dominantes; motivo por el cual muchos teóricos sociales consideran a la escuela como uno de los principales mecanismos de reproducción y mantenimiento de las ideologías dominantes (Althusser, 1971; Apple, 1982). Lo que sí es claro es que ya sea para la reproducción o para la emancipación, todas las polémicas importantes surgidas en torno a las reformas fundamentales en el país, reflejan la importancia que los grupos políticos conceden a la escuela como "germen de la cultura política".

Así, se considera que la enseñanza del portugués estaría más volcada a intereses políticos que al reconocimiento y valorización de que el portugués es la lengua materna ${ }^{35}$ de una gran porción de la población del país, y aún más, se apuesta a un tratamiento de la lengua que actúe como sustituta de la variedad hablada en frontera ${ }^{36}$. Aun así el tema en cuestión trata de cómo

35 La lengua materna es entendida como la primera lengua que los hablantes adquieren en el ámbito familiar. Por oposición a esta está la segunda lengua, considerada como la que se adquiere o aprende posteriormente a la materna (Programa de Educación Inicial y Primaria; Pág. 54).

36 Disponible con mayor profundidad en Barrios, G. (2008)”Discursos hegemónicos y representaciones lingüísticas sobre lenguas en contacto y de contacto: español, portugués y portuñol fronterizos". En: D. Da Hora y R. Marques de Lucena (orgs)Política Lin- 
O "SABER" DO DISCURSO HEGEMÔNICO COMO MANIPULADOR DAS MENTES E FORMADOR DE IDENTIDADES- A REALIDADE DA IMPLEMENTAÇÃO DO PORTUGUÊS EM REGIÃO DE FRONTEIRA

se ha ido implementando esta enseñanza, ya que desde los ámbitos formales, se ha proporcionado una escasa formación a los docentes (respecto a la lengua- portugués) y pareciera que esta formación se ha ido consolidando "en la marcha", así como han ido cambiando los discursos. En palabras de Henry Giroux:

(... ) no hay ya más caminos seguros. Sólo hay posibilidades efímeras para que pensemos a través del pasado, para que examinemos las historias sedimentadas que constituyen lo que somos y nos podamos insertar en el presente para luchar por una sociedad mejor.

(1992)

\section{DESARROLLO}

\subsection{Breve histórico}

Históricamente, el sistema educativo uruguayo no consideró la situación lingüística tan compleja, hasta que en 2003 , se implementa la enseñanza bilingüe en escuelas fronterizas. La escuela enfocaba esta realidad como un gran problema a resolver, "corrigiendo" las hablas locales. Así, a lo largo de más de un siglo, la institución escolar estigmatizó la variedad dialectal prohibiendo su uso en el ámbito educativo y hasta recibiendo la connotación de "problema de aprendizaje" de los alumnos. Se desconocían, en este contexto, los beneficios del bilingüismo, ignorándose la importancia social y psicológica del respeto por la lengua materna del niño

En este sentido, se trató la enseñanza de la Lengua con un enfoque nacionalista, luego purista y finalmente, se ha regido según las pautas determinadas a nivel mundial y regional en relación a la globalización.

\subsection{CONFORMACIÓN DEL ESCENARIO EDUCATIVO Y LINGÜÍSTICO}

A través de un análisis de la situación de la enseñanza del portugués en contexto de frontera, podemos decir que hemos pasado por un discurso y una enseñanza con apuesta a lo "nacional”, luego "contra el portugués",

guística na América Latina. João Pessoa. Ideia/Editora Universitária.79-103. 
purista y finalmente una apuesta a la formación de una cultura que responda a los objetivos de la globalización.

Es posible desde un análisis de los discursos percibir que no existe actualmente conferencia nacional o internacional donde la problemática de la globalización no esté presente. Considerando la complejidad de su fenómeno - las consecuencias de su extensividad en el planeta y la inter penetrabilidad de sus efectos que es generada por esta nueva configuración mundial denominada "sociedad en red" - cabe resaltar las contradicciones que esta situación impone a la cuestión de la lengua en zona de frontera dada principalmente las características de conformación geográfica e histórica de la región.

A primera vista, parece un ejercicio de imposibilidad, tratar de establecer un denominador entre lo que se discursa desde los discursos hegemónicos y lo que realmente ocurre en este contexto; pero es posible percibir como una vez más la institución escolar ha contribuido a formar al ciudadano aunque sin una clara reflexión respecto a qué precio esto se hizo y se hace posible.

Desde el año 2003 se ha incursionado en el funcionamiento del Programa de Educación Bilingüe (Portugués) en escuelas de Rivera, así como en otros departamentos de frontera con Brasil, expandiéndose el universo de escuelas partícipes en este proyecto, bajo dos modalidades: por Contenidos Curriculares y por Inmersión Dual (en Escuelas de Tiempo Completo). A pesar de ello solamente comenzó a funcionar el profesorado de portugués en este departamento (y otro grupo en Montevideo) recién en el año 2009, llegando a culminar su formación la primera generación en 2012.

La carrera habilita (según lo establece el Programa del Curso) a los docentes a desempeñarse en Educación Primaria como en Educación Secundaria (donde existen apenas algunos grupos con la disciplina en la Escuela Técnica y en el CLE- Centro de Lenguas Extranjeras).

Por otro lado, desde la definición dada en el Programa escolar (2008) respecto a Lengua Materna, Segundas Lenguas y Lenguas Extranjeras, pareciera ser un tanto confusa la consideración de la diversidad desde cómo es plasmada desde las políticas lingüísticas y los discursos, ya que la enseñanza del portugués aparece en dicho documento oficial en el apartado que se titula "Segundas Lenguas y Lenguas Extranjeras" y el contexto contemplado dentro del Programa de Segundas Lenguas de Educación Primaria es la situación de frontera. Siendo así la enseñanza del portugués debería enseñarse como primera lengua o lengua materna. Más aún se hace presente en dicho 
O "SABER" DO DISCURSO HEGEMÔNICO COMO MANIPULADOR DAS MENTES E FORMADOR DE IDENTIDADES- A REALIDADE DA IMPLEMENTAÇÃO DO PORTUGUÊS EM REGIÃO DE FRONTEIRA

documento explícitamente que la enseñanza del portugués se hará en "portugués estándar" 37 , es decir la "norma culta" que para nada tendrá que ver con la variedad hablada por gran parte de la población fronteriza.

Más aún, la mayoría de los docentes ha incursionado a trabajar dentro del Programa de Segundas Lenguas con un conocimiento básico de la Lengua o nulo; posterior a ello algunos docentes han buscado la mejora de sus conocimientos en portugués y de gran modo esto se debe a la gran demanda de docentes para cubrir los cargos de Docente de Portugués en escuelas del Departamento y la escasa o tardía capacitación brindada por el Sistema Educativo.

Esta realidad muestra claramente la dicotomía que existe entre lo que se discursa o se pretende a nivel político y económico y la realidad de cómo ello ocurre en la práctica.

Cabría preguntarnos donde han aprendido el tan anhelado "portugués estándar" o como es llamado por muchos docentes "portugués correcto" y cuál será el producto de esta enseñanza ya que la "variedad del portugués hablada en el departamento", Ilamada por muchos autores como DPU (Dialectos Portugueses del Uruguay), o "portuñol" es la lengua materna de muchos docentes y es la escuela es el ámbito donde debería el alumno verse expuesto a la lengua y aprenderla.

\subsection{APUESTAS A LA “NUEVA" REALIDAD EDUCATIVA}

Muchos fueron los estudios y aportes a este escenario educativo (Brovetto, C.; Geymonat, J.; Brian, N. y los aportes de Ana Maria Carvalho; Documentos e informes Técnicos de la Educación Pública y estudios realizados en la región de frontera por Behares,L. y Barrios,G.).

La cuestión en el momento pasa por lo menos por dos cuestiones fundamentales: brindar una Formación docente acorde a los nuevos requisitos y estructurar una organización inter sistémica que permita la secuencia educativa de los alumnos que culminan sexto año escolar, pues la continuidad

37 Ver Programa de Educación Inicial y Primaria (Año 2008), en la que aparece como motivación para introducir la enseñanza del portugués, deriva de reconocer y respetar la identidad lingüística de la población escolar del contexto fronterizo, pero además la necesidad de comunicación a nivel regional dada la proximidad con Brasil. Aun así aparece escrito con letra en negrita: "Lo anterior no significa que la variedad local del portugués sea la lengua de instrucción, ya que por el contrario, ese será el papel del portugués estándar..." (pág. 55). 
del estudio de la lengua solo lo ofrece el CLE (Centro de Lenguas Extranjeras, contando en Rivera con un único centro, limitados cupos y siendo una formación no obligatoria).

\subsection{EN VISTA DE LOS CAMBIOS}

El Programa de Segundas Lenguas y Lenguas Extranjeras (CEIP Consejo de Educación Inicial y Primaria), tuvo comienzo en escuelas de Tiempo Completo de Artigas y Rivera (año 2003), no contando con docentes con formación específica en la lengua, cubriéndose los cargos con maestros que rendían una prueba oral y escrita y poseían en algunos casos cursos (en su mayoría privados) en Portugués.

En el año 2008, la enseñanza del Portugués se instrumenta como parte del Programa Escolar de Segundas Lenguas y Lenguas Extranjeras (Currículum Oficial) y tiene como objetivos principales:

*promover el bilingüismo y el bidialectalismo;

* respetar y valorizar la variedad lingüística fronteriza;

* desarrollar la oralidad y la escritura en español y en portugués estándares;

*adquirir conocimientos en diferentes áreas del currículo escolar;

*mejorar los resultados académicos de los alumnos;

* promover actitudes positivas entre la escuela y la comunidad.

Recién en el año 2009 es implementado el curso de profesorado de Portugués, teniendo como cede Rivera y Montevideo.

En 2013, transcurridos diez años del comienzo de la enseñanza del portugués en escuelas de frontera, el CEIP hace un primer llamado para proveer cargos efectivos de docentes de Portugués LE (Lengua Extranjera), con una carga horaria de 20 hs.

Aun así, es motivo de reflexión, que educación secundaria no mantenga una secuencia en la enseñanza del portugués en su currículum, ya que la nueva Ley General de Educación ( $\mathrm{N}^{\circ}$ 18.437), promulgada en 2008, incluye una breve, pero densa en contenidos, referencia a las cuestiones lingüísticas. En esta ley, la "educación lingüística" es una de las nueve líneas transversales a contemplar en el Sistema Nacional de Educación:

La educación lingüística tendrá como propósito el desarrollo de las competencias comunicativas de las personas, el dominio de la lengua escrita, el respeto de las variedades lingüísticas, la reflexi- 
O "SABER" DO DISCURSO HEGEMÔNICO COMO MANIPULADOR DAS MENTES E FORMADOR DE IDENTIDADES- A REALIDADE DA IMPLEMENTAÇÃO DO PORTUGUÊS EM REGIÃO DE FRONTEIRA

ón sobre la lengua, la consideración de las diferentes lenguas maternas existentes en el país (español del Uruguay, portugués del Uruguay, lengua de señas uruguaya) y la formación plurilingüe a través de la enseñanza de segundas lenguas y lenguas extranjeras. (MEC, 2008: Capítulo VII, Art. 40, Inc.5)

En este párrafo, destinado a la "educación lingüística", queda claro que la ley reconoce la condición heterogénea en materia lingüística de la sociedad uruguaya y la integra a los propósitos de la educación como dimensión a considerar en lo educativo. Puede interpretarse que hace referencia a la heterogeneidad que mencionábamos anteriormente.

Se menciona explícitamente al portugués del Uruguay como lengua materna, existente en el país. De modo concomitante al reconocimiento de la heterogeneidad lingüística propia, se expresa la intención de educación plurilingüe:

(... ) En lo que respecta específicamente a la enseñanza de Portugués, se prevé su introducción en distintos niveles dependiendo del contexto. En las escuelas de zona fronteriza bilingüe o con presencia de Portugués se universalizará la enseñanza de Portugués desde la Educación Inicial. En el resto del país, se incorporará en los últimos años del tramo escolar $\left(5^{\circ}\right.$ y $6^{\circ}$ año) y en los primeros años del Ciclo Básico de Enseñanza Media. A su Vez, está previsto extender la oferta de enseñanza de portugués a través de los Centros de Lenguas Extranjeras (CLE), que se abrirán a los estudiantes de Bachillerato en el futuro, a los docentes del sistema en general"(Cita extraída de: http://www.cfe.edu.uy/index.php/ planes-y-programas/planes-vigentes-para-profesorado/44-planes-y-programas/profesorado-2008/396-portugues).

Mismo así, siempre se hace referencia a la "lengua estándar".

\subsection{COMO INCIDE EN LOS ACTORES SOCIALES DEL CONTEXTO DE FRONTERA ESTA REALIDAD}

Según el autor Marcos Bagno (1995), solamente cuando reflexionamos sobre nuestra realidad, es posible apreciar que el modo que nos auto percibimos está influenciado principalmente por los medios de comunicación (que a su vez, transmiten los discursos y representaciones que políticamente importan transmitirse).

Los DPU sufren con la discriminación apoyada históricamente por as- 
pectos políticos, sociales, culturales, y que fueron caracterizando "perfiles", los que serían los modos de ser y actuar de los ciudadanos en este contexto.

Aún en nuestro contexto, es creíble por muchos, que la norma culta debe ser el principal objeto de enseñanza-aprendizaje. Pero por diferentes factores, sabemos que e reservada a una minoría.

En vista de las consideraciones de Bagno expuestas anteriormente, esto puede acarrear por lo menos tres problemas básicos: el analfabetismo (una cualidad injustificada de personas analfabetas o alfabéticas funcionales); las personas plenamente alfabetizadas no poseen el hábito de leer y escribir, y cuando lo hacen, no cultivan, ni desenvuelven sus habilidades en el nivel de la norma culta; el dilema de la norma culta usada por la tradición gramatical que no corresponde a la lengua hablada por las personas cultas de hoy, y si a los ideales lingüísticos del Portugués “ideal” (Portugal), haciendo a la norma culta real distante de la norma culta ideal.

\subsection{UNA ALTERNATIVA DE CAMBIO}

Es necesario reconocer que el pre concepto lingüístico continua muy fuerte, y nada va a cambiar en la sociedad en la cual estamos insertos se no ocurren significativas modificaciones en el "ideario social y popular". Pero es posible tomar en manos algunas actitudes contra la situación que se explicita anteriormente.

Según Bagno M. (1995), es necesario que nos hagamos críticos e investigadores de nuestro propio conocimiento lingüístico, dejando de lado la actitud repetitiva y reproductora, formándonos e informándonos.

En segundo lugar, se hace crucial una criticidad en lo que refiere a nuestra práctica diaria.

Como un tercer punto, tener una conciencia de que las ciencias evolucionan, así como la ciencia del Lenguaje. Necesitamos actualizarnos y hasta innovar.

Una cuarta actitud, seria asumir una nueva postura con base en lo que el autor llamó "DEZ CISÕES", porque representa un corte con todas las ultrapasadas normas de la gramática tradicional. Son ellas:

(... ) concientizarse de que todo hablante nativo de una lengua es un usuario competente de esa lengua, dominándola por completo; aceptar la idea de no existe error en lengua, apenas diferencias o alternativas posibles; no confundir error en la lengua con error en ortografía, que es artificial e que puede sufrir modificaciones, 
O "SABER" DO DISCURSO HEGEMÔNICO COMO MANIPULADOR DAS MENTES E FORMADOR DE IDENTIDADES- A REALIDADE DA IMPLEMENTAÇÃO DO PORTUGUÊS EM REGIÃO DE FRONTEIRA

contrario a la lengua, que es natural; reconocer que todo lo que la gramática tradicional llama de error, es en la verdad un fenómeno perfectamente explicado, si la mayoría de los hablantes usa una norma que difiere de la tradicional, es porque ya existe una regla que se sobrepone a la antigua; aceptar que toda lengua cambia, lo que es visto hoy como "cierto", ya fue "error" en el pasado y así sucesivamente; concientizarse que la lengua no tiene un referente de buena o mala, ella apenas sigue su curso y su evolución; respetar la variedad lingüística de cualquier persona; entender que la lengua permeabiliza todo, y nosotros somos la lengua que hablamos, es ella quien moldea nuestra forma de ver el mundo y nuestro modo de ver el mundo moldea la lengua que hablamos. Finalmente: enseñar bien y para el bien, respetando el conocimiento del otro, valorizando lo que él ya sabe del mundo y de la vida, reconociendo en la lengua que habla su propia identidad como ser humano, siempre enriqueciéndolo y elevando su autoestima.

Aunque su trabajo analiza la situación del portugués hablado en Brasil comparativamente con el portugués de Portugal, se adecúan perfectamente sus apreciaciones al contexto de frontera.

\section{CONSIDERACIONES FINALES}

Es posible afirmar que en el contexto de frontera cobra relevancia un aspecto que se ha convertido en preocupación en diferentes países del mundo, y es la relación entre el proceso administrativo burocrático de la planificación educativa y los intereses, y las necesidades de los miembros y clientes del sistema educativo (padres, alumnos, maestros). El problema principal y más que nada real, es como relacionar los intereses y las necesidades populares con la estructura de toma de decisiones que casi por definición es política, elitista y está más o menos alejada de las masas. Esto pareciera verse claramente en el contexto fronterizo.

Barrios, G. (2008), en su trabajo sobre lenguas en contacto y de contacto, sostiene que:

\footnotetext{
"la tradicional política lingüística del Estado uruguayo ha sido sostenida por un discurso valorizador de la lengua española y desacreditador del portugués, que tiene su origen en el reconocimiento expreso del problema fronterizo" (pàg.3).
}

El portugués fronterizo aparece entonces como una mezcla que debe ser sustituida por un "español correcto". 
Así también podría considerarse el discurso actual: ya no se discursa solamente un "español correcto", sino un "portugués correcto". Incluso en los mejores discursos se extrae una opinión positiva respecto a su enseñanza en tanto sea operacional para "corregir" o incluso eliminar el uso de una cierta variedad lingüística (es decir, un programa de enseñanza de lenguas de tipo sustractivo). Es decir, se apuesta a una propuesta a la "corrección" de los elementos supuestamente erróneos característicos de estas formas “mezcladas" de una lengua y otra (Español y Portugués). En este contexto tan complejo como lo es la situación de frontera, cabría tal vez analizar la función de la pragmática en el lenguaje y en su adquisición. Ésta consiste en el estudio de cómo se emplea el habla para lograr fines sociales.

Se sabe de la implicancia de la Educación como medio para el logro de fines que determinada sociedad considera válidos en un momento histórico determinado. Pero más que nunca, cobra sentido destacar la importancia de la responsabilidad y el compromiso de cuestionarla, desde nuestros roles.

El Sistema Educativo ha ido acompañando los lineamientos de las políticas educativas y lingüísticas, donde las distintas alternativas encuentran su síntesis en el currículum real, articulándose diferentes transposiciones didácticas, es decir, el conjunto de procedimientos y mediaciones a través de las cuales el conocimiento erudito se convierte en conocimiento aprendido. En este punto se entrelaza el currículum prescripto, la propuesta editorial y la cultura pedagógica de los docentes.

En vista de esto es posible decir que existe un intento por implementar una propuesta en vista del "escenario educativo" que se estructura y así por parte de los docentes, una manifiesta reflexión acerca del tema, pero al mismo tiempo, existe una gran dificultad en poder sostener un discurso en vista de lo que se piensa y se hace y hasta de analizar críticamente lo que se "piensa" en vista de los discursos hegemónicos y las representaciones que han elaborado.

En determinados momentos se discursa atención a la diversidad, pero no existe una real contemplación de la variedad lingüística que trae el alumno (considerada una mezcla), reflejo de los discursos hegemónicos que certifican que la enseñanza de ambas lenguas (Español y Portugués) se harán en la "lengua estándar".

En este complejo contexto no se encuentran solo docentes de la región de frontera, sino todos los involucrados de la tarea educativa y más aún los propios niños, que en ese ir y venir continúan su aprendizaje, dándose lugar 
O "SABER" DO DISCURSO HEGEMÔNICO COMO MANIPULADOR DAS MENTES E FORMADOR DE IDENTIDADES- A REALIDADE DA IMPLEMENTAÇÃO DO PORTUGUÊS EM REGIÃO DE FRONTEIRA

al desarrollo de sus posibilidades o limitaciones.

\section{REFERÊNCIAS}

ANEP.CODICEN.CEIP- Programa de Educación Inicial y Primaria. Montevideo. Uruguay((2008).

BAGNO, Marcos .A luta desigual. Mito vs. realidade nos livros didáticos de língua portuguesa. Dissertação de Mestrado, Recife, Programa de Pós-graduação em Letras e Linguística, Universidade Federal de Pernambuco, mimeo. (1995).

BARRIOS, G. "Discursos hegemónicos y representaciones lingüísticas sobre lenguas en contacto y de contacto: español, portugués y portuñol fronterizos". En: D. Da Hora y R.Marques de Lucena (orgs) Política Lingüística na América Latina. João Pessoa, Ideia/ Editora Universitária, 2008. Pág. De la 79 a la 103.

BEHARES, L. E. Portugués del Uruguay y educación fronteriza En: Brovetto, Geymonat y Brian (eds) Portugués del Uruguay y Educación Bilingüe. ANEP, Montevideo (2007).

BROVETTO, C., J. Geymonat y N. Brian. Una experiencia de educación bilingüe español portugués en escuelas de la zona fronteriza En: Brovetto, Geymonat y Brian (eds) Portugués del Uruguay y Educación Bilingüe. ANEP, Montevideo (2007) .

CARVALHO, A. M. Diagnóstico sociolingüístico de comunidades escolares fronterizas en el norte de Uruguay En: Brovetto, Geymonat y Brian (eds) Portugués del Uruguay y Educación Bilingüe. ANEP, Montevideo (2007).

DeLEX (Departamento de Lenguas Extranjeras)- ANEP- CODICEN- Dirección de Formación y Perfeccionamiento Docente (2008). Disponible desde internet en http://www.cfe. edu.uy/index.php/planes-y-programas/planes-vigentes-para-profesorado/44-planes-y-programas/profesorado-2008/396-portugues)

Educación bilingüe de frontera y políticas. Disponible desde internet en http://www. scielo.br/pdf/pp/v21n3/v21n3a03

LÉMEZ, R. "Gobierno y Administración de la Educación en el Uruguay". Un análisis sociopolítico del "campo" de la Administración Educativa: características de su constitución y consolidación. Documento de trabajo. PRIE.UCUDAL. Cap. I.Aspectos de Teoría. Pág.9 a 33. Montevideo (2001).

“Análisis Sociopolítico de la Educación en el Uruguay. Doc.De Trabajo. Programa Educación IU-CLAEH. Montevideo, Noviembre de 2006.

RECEBIDO EM: 16/05/2015

ACEITO PARA PUBLICAÇÃO: 02/07/2015 


\title{
Rosario M. Acuña Flores
}

\begin{abstract}
Mtra de Ed. Inicial y Común. Prof. de Portugués. Especialización y Maestría en Didáctica de la Ed. Básica y Media. Post-graduación en Metodología de Enseñanza de la L.P y L. y Psicopedagogía Institucional. Docente de Portugués en Ed. Primaria y en el CeRP del Norte. Con estudios relacionados a la lengua en región de frontera. Actualmente integrante del NEISELF (Núcleo de Estudios Interdisciplinarios sobre Sociedad, Educación y Lenguaje en Frontera).
\end{abstract}


